\title{
Electron Monopole Duality in Quantum Hall Effect
}

\author{
Pawan Ku. Joshi, Praveen Singh Bisht, Om Prakash Singh Negi
}

Department of Physics, Kumaun University, S. S. J. Campus, Almora1(Uttarakhand), India.

Email: pj4234@gmail.com,ps_bisht123@rediffmail.com,ops_negi@yahoo.com

Received November 25 ${ }^{\text {th }}, 2010$; revised December 20 ${ }^{\text {th }}, 2010$; accepted December $29^{\text {th }}, 2010$.

\begin{abstract}
Starting from the duality between electric and magnetic field, we have made an attempt to discuss the quantum hall effect from the consideration of magnetic monopole in view of electron monopole duality. Starting from the dual dynamics of electric and magnetic charges, we have reformulated a consistent theory of quantum hall effect in presence of monopole. Speculating the existence of magnetic monopoles in magnetic materials (metals), we have accordingly modified the parameters; like drift velocity, current density, Hamiltonian and eigen values and eigen function for harmonic oscillator; resposible to examine the quantum Hall effect in metals.
\end{abstract}

Keywords: Hall Effect, Monopole, Quantum Mechanics

\section{Introduction}

The Hall Effect was discovered by Sir Edwin Hall [1] in 1879 while he was under graduate student in Johns Hopkins University. But at that time, even the electron was not experimentally discovered. Clear understanding had to wait until quantum mechanics came in to appearance. A hundred years later, the Hall effect was revived as a source of wonderful physics. In 1980, Klaus von Klitzing discovered [2] that two dimensional electron gas, at very low temperatures and strong magnetic fields, displays a remarkable quantization of the Hall conductance. Namely, the graph of the Hall conductance as the function of the magnetic field, is a staircase function, where the value of the Hall conductance at the plateaus is, to great accuracy, an integer multiple of $e^{2} / h=1 /(25812.807572 \Omega)$. This discovery led to superior standards of resistance and von-Klitzing was awarded the Nobel Prize in 1985 for his discovery. In a 1981 Robert Laughlin put forward [3] an argument for the quantization of the Hall conductance. This argument played a seminal role in the development of the theory of the Integer Hall effect. On the other hand, the lack of symmetry between electric and magnetic fields is one of the oldest puzzles in physics. One of the biggest unresolved questions in theoretical physics is that associated with the quantization of electric charge, i.e. why the observed electric charges in all the electrically charged matter is an integer multiple of a "fundamental charge" ' $e$ ', the electron charge. Why is it possible to iso- late positive and negative electric charges, but not north and south magnetic poles? P. A. M. Dirac [4] introduced the idea of magnetic charge (monopole) ' $g$ ' in the universe to answer this question. As a matter of fact, the quantum dynamics of a particle with electric charge ' $e$ ' under the influence of the magnetic field generated by such particle is well defined if the well known Dirac Quantization condition [4] 'eg $=1 / 2 n \hbar c$ ', (where $n \in Z$ is an integer) is satisfied. But unfortunately all experimental searches [5] to discover the magnetic monopoles have been found to be negative and proved their existence fruitless. However, a group of physicists has been claimng [6] now a days the possibility of indirect evidences of magnetic monopoles in the consnsed matter physics.. So, it is being speculated [7] that that magnetic materials may provide a new context for observing magnetic monopoles which plays an important role in condensed matter physics. While a magnetic monopole particle has never been conclusively observed, there are a number of phenomena [6,7] in condensed-matter physics where a material, due to the collective behavior of its electrons and ions, can show emergent phenomena that resemble magnetic monopoles in some respect. So, keeping in view the recent interests on monopole and their possible role to produce strong magnetic field resposible for quantum Hall effect, in this paper, we have made an attempt to investigate the consistent theory of classical and quantum Hall effect for which the magnetic 
field is produced due to the presence of magnetic monopole like electron produces electric field. Starting from the duality between electric and magnetic field, we have tried to study the theory of quantum hall effect from the consideration of magnetic monopole in view of electron monopole duality. Taking into account the dual dynamics of electric and magnetic charges, we have reformulated a consistent theory of quantum hall effect in presence of monopole. Speculating the magnetic monopoles in magnetic materials, we have accordingly, modified the parameters; like drift velocity, current density, Hamiltonian, eigen values and eigen function for harmonic oscillator; resposible to examine the quantum Hall effect in metals.

\section{Dual Electrodynamics}

The concept of electromagnetic (EM) duality has been receiving much attention $[8,9]$ in gauge theories, field theories, Supersymmetry and super strings. Duality invariance is an old idea introduced a century ago in classical electromagnetism for the following Maxwell's equations in vacuum (using natural units $c=\hbar=1$, space-time four-vector $\left\{x^{\mu}\right\}=(t, x, y, z),\left\{x_{\mu}\right\}=\eta_{\mu v} x^{\mu}$ and $\left\{\eta_{\mu v}=+1,-1,-1,-1=\eta^{\mu v}\right\}$ through out the text) [10],

$$
\begin{array}{ll}
\vec{\nabla} \cdot \vec{E}=0 ; & \vec{\nabla} \cdot \vec{H}=0 ; \\
\vec{\nabla} \times \vec{E}=-\frac{\partial \vec{H}}{\partial t} ; & \vec{\nabla} \times \vec{H}=\frac{\partial \vec{E}}{\partial t} ;
\end{array}
$$

as these were invariant not only under Lorentz and conformal transformations but also invariant under the following duality transformations,

$$
\begin{aligned}
& \vec{E} \Rightarrow \vec{E} \cos \vartheta+\vec{H} \sin \vartheta ; \\
& \vec{H} \Rightarrow-\vec{E} \sin \vartheta+\vec{H} \cos \vartheta ;
\end{aligned}
$$

where $\bar{E}$ and and $\bar{H}$ are respectively the the electric and magnetic fields. For a particular value of $\vartheta=\pi / 2$, Equations (2) reduces to

$$
\vec{E} \rightarrow \vec{H} ; \vec{H} \rightarrow-\vec{E} ;
$$

which can be written as

$$
\left(\begin{array}{l}
\vec{E} \\
\vec{H}
\end{array}\right) \Rightarrow\left(\begin{array}{cc}
0 & 1 \\
-1 & 0
\end{array}\right)\left(\begin{array}{l}
\vec{E} \\
\vec{H}
\end{array}\right) .
$$

Let us introduce a complex vector $\bar{\Psi}=\bar{E}+i \bar{H}$ $(i=\sqrt{-1})$ so that the Maxwell's Equations (1) be written as

$$
\vec{\nabla} \cdot \vec{\Psi}=0 ; \quad \vec{\nabla} \times \vec{\Psi}=i \frac{\partial \vec{\Psi}}{\partial t} ;
$$

which is also invariant under following duality transformations

$$
\vec{\Psi} \rightarrow \exp (i \vartheta) \vec{\Psi} .
$$

The duality symmetry is lost if electric charge and current source densities enter to the conventional Maxwell's equations given by

$$
\begin{aligned}
& \vec{\nabla} \cdot \vec{E}=\rho_{e} ; \quad \vec{\nabla} \times \vec{H}=\vec{j}+\frac{\partial \vec{E}}{\partial t} ; \\
& \vec{\nabla} \cdot \vec{H}=0 ; \quad \vec{\nabla} \times \vec{E}=-\frac{\partial \vec{H}}{\partial t} ;
\end{aligned}
$$

where $\left\{j^{\mu}\right\}=\left(\rho_{e}, \vec{j}\right)$ is described as four-current source density and symbol $e$ is used for electric charge. Consequently, Maxwell's equations may be solved by introducing the concept of vector potential in either two ways. The conventional choice has been used as

$$
\vec{E}=-\frac{\partial \vec{A}}{\partial t}-\operatorname{grad} \varphi ; \vec{H}=\vec{\nabla} \times \vec{A}
$$

where $\left\{A^{\mu}\right\}=(\varphi, \vec{A})$ is denoted as the four potential. Accordingly, the second pair of the Maxwell's Equations (7) becomes kinematical identities. So the dynamics is contained in the first pair. Equation (5) is now modified as

$$
\vec{\nabla} \cdot \vec{\Psi}=\rho_{e} ; \quad \vec{\nabla} \times \vec{\Psi}=i \frac{\partial \vec{\Psi}}{\partial t}+i \vec{j} ;
$$

which is no more invariant under duality transformations (6). Here if we may consider the another alternative way to write

$$
\vec{H}=-\frac{\partial \vec{B}}{\partial t}-\operatorname{grad} \phi ; \quad \vec{E}=-\vec{\nabla} \times \vec{B} ;
$$

by introducing another potential $\left\{B^{\mu}\right\}=(\phi, \vec{B})$, we see that source free Maxwell's Equations (1) are unchanged but but Maxwell's Equations (7) are changed as

$$
\begin{array}{ll}
\vec{\nabla} \cdot \vec{E}=0 ; & \vec{\nabla} \times \vec{H}=\frac{\partial \vec{E}}{\partial t} ; \\
\vec{\nabla} \cdot \vec{H}=\rho_{m} ; & \vec{\nabla} \times \vec{E}=-\vec{k}-\frac{\partial \vec{H}}{\partial t} ;
\end{array}
$$

where $\left\{k^{\mu}\right\}=\left(\rho_{m}, \vec{k}\right)$. Here, the first pair becomes kinematical whereas the dynamics is contained in the second pair. Equation (11) may also be obtained if we apply the transformations (3-4) along with the following duality transformations for potential and current i.e.

$$
\begin{aligned}
& A^{\mu} \rightarrow B^{\mu} ; B^{\mu} \rightarrow-A^{\mu} \Leftrightarrow\left(\begin{array}{l}
A^{\mu} \\
B^{\mu}
\end{array}\right) \Rightarrow\left(\begin{array}{cc}
0 & 1 \\
-1 & 0
\end{array}\right)\left(\begin{array}{l}
A^{\mu} \\
B^{\mu}
\end{array}\right) ; \\
& j^{\mu} \rightarrow k^{\mu} ; k^{\mu} \rightarrow-j^{\mu} \Leftrightarrow\left(\begin{array}{c}
j^{\mu} \\
k^{\mu}
\end{array}\right) \Rightarrow\left(\begin{array}{cc}
0 & 1 \\
-1 & 0
\end{array}\right)\left(\begin{array}{l}
j^{\mu} \\
k^{\mu}
\end{array}\right) .
\end{aligned}
$$


So, we may identify the potential $\left\{B^{\mu}\right\}=(\phi, \vec{B})$ as the dual potential and the current $\left\{k^{\mu}\right\}=\left(\rho_{m}, \vec{k}\right)$ is used as the dual current. The symbol $m$ is written for magnetic (dual of electric) case. Correspondingly, the differential Equations (11) are identified as the dual Maxwell's equations and accordingly one can develop the dual electrodynamics. Thus, the concept of electromagnetic duality in the Maxwell's equations establishes the connection between electric and magnetic charge as,

$$
e \rightarrow g ; g \rightarrow-e \Leftrightarrow\left(\begin{array}{l}
e \\
g
\end{array}\right) \Rightarrow\left(\begin{array}{cc}
0 & 1 \\
-1 & 0
\end{array}\right)\left(\begin{array}{l}
e \\
g
\end{array}\right)
$$

where $g$ is described as the dual electric charge (charge of magnetic monopole). Hence we may recall the dual electrodynamics as the dynamics of pure magnetic monopole and the corresponding physical variables associated there are described as the dynamical quantities of magnetic monopole.

The Lorentz force equation of motion for a dual charge (i.e magnetic monopole) may now be written from the duality Equations (3) and (13) as

$$
\frac{d \vec{p}}{d \tau}=\overrightarrow{F_{m}}=m \overrightarrow{\ddot{x}}=g(\vec{H}-\vec{v} \times \vec{E})
$$

where $\vec{p}=m \overrightarrow{\dot{x}}=m \vec{v}$ is the momentum, and $\vec{f}$ is a force acting on a particle of charge $g$, mass $m$ and moving with the velocity $\vec{v}$ in electromagnetic fields.

\section{Hall Effect in Presence of Monopoles}

The QHE is a manifestation of quantum mechanics observable at macro- scopic scales. In order to illustrate the role of magnetic monopole in classical and Quantum Hall Effect, let us start with the Lorentz force Equation (14). It is to be noted that for the case of pure monopole, we may assume the magnetic field as stationary and electric field is obtained for moving magnetic particle containing monopole. If the volume charge density of a magnetic monopole is $\rho_{m}$, then the magnetic current density $\bar{k}$ is described as

$$
\vec{k}=\rho_{m} \vec{v}
$$

and accordingly the continuity equation reduces to

$$
\vec{\nabla} \cdot \vec{k}+\frac{\partial \rho_{m}}{\partial t} .
$$

So the Biot-Savart law in presence of magnetic monopole charge is associated with electric field and is thus defined as

$$
\vec{E}(r)=\frac{1}{4 \pi \varepsilon_{0}} \int \frac{I_{m} \times \hat{r}}{r^{2}} d l
$$

where $I_{m}$ is a magnetic current, $\varepsilon_{0}$ is permittivity of free space. So, the Lorentz-Drude theory is modified for magnetic conductivity and gives rise to the following expression for drift velocity as

$$
\overrightarrow{v_{d}}=-g \vec{H} \frac{\tau}{m}
$$

where $\tau$ is the relaxation time and $m$ is the mass of magnetic monopole. So, the magnetic current density is modified as

$$
\vec{k}=-n g \overrightarrow{v_{d}}=n g\left(\frac{g \tau}{m}\right) \vec{H}=\sigma_{0} \vec{H}
$$

where $\sigma_{0}=n g^{2} \tau / m$. In the study state case the Lorentz force Equation (14) is vanishing and thus leads to

$$
\vec{H}=\frac{\vec{v} \times \vec{E}}{c} .
$$

Hence, the current (15) is described as

$$
\vec{k}=\sigma_{0} \frac{\vec{v} \times \vec{E}}{c} .
$$

Thus, the expressions for steady state behavior of conductivity and resistivity become

$$
\sigma=\left(\begin{array}{cc}
\sigma_{x x} & \sigma_{x y} \\
\sigma_{y x} & \sigma_{y y}
\end{array}\right) ; \rho=\left(\begin{array}{cc}
\rho_{x x} & \rho_{x y} \\
\rho_{y x} & \rho_{y y}
\end{array}\right) .
$$

Hence, we get

$$
\vec{k}=\sigma \vec{H} .
$$

Accordingly, we may modify the drift velocity for magnetic monopole. Thus the classical Hall effect for two dimensional case in presence of monopoles describes

$$
\begin{aligned}
& \sigma_{0} H_{x}=j_{x}-\omega_{c} \tau j_{y} ; \\
& \sigma_{0} H_{y}=j_{y}+\omega_{c} \tau j_{x} ;
\end{aligned}
$$

where $\omega_{c}$ is the cyclotron frequency given by

$$
\omega_{c}=\frac{g E}{m c} .
$$

So, we can easily get

$$
\rho_{x x}=\rho_{y y}=\frac{1}{\sigma_{0}},-\rho_{x y}=\rho_{y x}=\frac{\omega_{c} \tau}{\sigma_{0}}
$$

and

$$
\begin{aligned}
& \sigma_{x x}=\sigma_{y y}=\frac{\sigma_{o}}{1+\left(\omega_{c} \tau\right)^{2}} ; \\
& \sigma_{x y}=-\sigma_{y x}=\frac{\sigma_{o} \omega_{c} \tau}{1+\left(\omega_{c} \tau\right)^{2}}
\end{aligned}
$$

Hence, the relation between conductivity and resistivity is described as 


$$
\sigma_{x x}=\frac{\rho_{x x}}{\rho_{x x}^{2}+\rho_{x y} 2}, \sigma_{x y}=-\frac{\rho_{x y}}{\rho_{x x}^{2}+\rho_{x y}^{2}} .
$$

for classical Hall effect. In quantum mechanics, the Hamiltonian is ( $\mathbb{H}$ along the $X$-direction)

$$
\mathbb{H}=\frac{1}{2 m}\left(p-\frac{g B}{c}\right)^{2}+g H_{x} .
$$

Now we choose the Landu gauge in which the vector potential is independent of $Y$ - coordinate as

$$
A=\left(0, E_{x}, 0\right) \text {. }
$$

Let us take a wave function which has a plane-wave dependence on the $Y$ - coordinate as

$$
\psi(x, y)=e^{-i k_{y} y} \phi(x)
$$

and substituting the Equation (31) into the Schroedinger equation, we get

$$
\left[-\frac{\hbar}{2 m} \frac{d^{2}}{d x^{2}}+\frac{1}{2} m \omega_{c m}^{2}\left(x-I_{c}^{2} k_{y}\right)^{2}+g H x\right] \phi(x)=\varepsilon \phi(x)
$$

where $I_{c}$ is the classical cyclotron orbit radius .

$$
I_{c}=\left(\frac{\hbar c}{g H}\right) \text {. }
$$

So, the eigan values and eigan states are described as

$$
\begin{gathered}
\varepsilon_{i}(E)=\left(i+\frac{1}{2}\right) \hbar \omega_{c}+g H\left(I_{c}^{2} k_{y}-\frac{g H}{2 m_{c} \omega_{c}^{2}}\right) \\
\psi_{i}(x, y)=e^{\left(-i k_{y} y\right)} \exp \left[-\left(x-x_{0}\right)^{2} / 2 I_{c}^{2}\right] H_{i}\left[\frac{\left(x-x_{0}\right)}{I_{c}}\right]
\end{gathered}
$$

where $x_{0}=I_{c}^{2} k_{y}-\frac{g E}{m \omega_{c}^{2}}$.

\section{Summary and Conclusions}

The precision of the quantization in the Hall effect is remarkable in that it takes place in systems that are imprecisely characterized on the microscopic scale. Different samples may have dierent distributions of impurities, different geometry and different concentrations of electrons. Nevertheless, whenever their Hall conductances are quantized, the quantized values mutually agree with great precision. The quantum Hall effect may also be interpreted [11] as a measurement of the fine structure constant so that the Hall conductance may have topological signicance. Since magnetic monopoles have the topological origin [12] and the quantum Hall effect is described in terms of strong magetic field, we have dis- cussed here the theory of classical and quantum Hall effect in presence of magnetic monopole so that the magnetic field can be obtained directly instead of rotating an electric charge. In order to seek the existence of magnetic monopoles in condensed matter physics [6,7], particularly in case of Hall effect, in the foregoing analysis, we have discussed the manifestly covariant theory of magnetic monopoles and established the connections among the various parameters of classical and quantum Hall effect in terms of electron monopole duality invariance.

\section{Acknowledgements}

One of us OPSN is thankful to Professor H. Dehnen, Universitt Konstanz, Fachbereich Physik, Postfach-M 677, D-78457 Konstanz, Germany for his hospitality at Universitt Konstanz. He is also grateful to German Academic Exchange Service (Deutscher Akademischer Austausch Dienst), Bonn for financial support under DAAD re-invitation programme.

\section{REFERENCES}

[1] E. H. Hall, "On a New Action of the Magnet on Electric Currents," American Journal of Mathematics, Vol. 2, 1879, pp. 287-292.

$$
\text { doi:10.2307/2369245 }
$$

[2] K. Von Klitzing, G. Dorda and M. Pepper, "New Method for High-Accuracy Determination of the Fine-Structure Constant Based on Quantized Hall," Physical Review Letters, Vol. 45, No. 6, 1980, pp. 494-497. doi:10.1103/PhysRevLett.45.494

[3] R. B. Laughlin, “Anomalous Quantum Hall Effect: An Incompressible Quantum Fluid with Fractionally Charged Excitations,” In: R. E. Prange and S. M. Girvin, Eds., Physical Review Letters, Vol. 50, No. 18, 1983, pp. 1395-1398.

[4] P. A. M. Dirac, "Quantised Singularities in the Electromagnetic Field,” Proceedings of Royal Society, London, 1931, pp. 60-72.

[5] P. B. Price, E. K. Shirk, W. Z. Osborne and L. S. Pinsky, "Evidence for Detection of a Moving Magnetic Monopole,” Physical Review Letters, Vol. 35, No. 8, 1975, pp. 487-490. doi:10.1103/PhysRevLett.35.487

[6] S. Zhang et al, "A Four-Dimensional Generalization of the Quantum Hall Effect,” Science, Vol. 294, No. 5543, 2001, pp. 823-828.

doi:10.1126/science.294.5543.823

[7] S. Sondhi, “An Experiment Based on Wien's Theory of Electrolytes Has Now Measured Its Value,” Nature, Vol. 461, No. 7266, 2009, pp. 888-889. doi:10.1038/461888a

[8] N. Seiberg and E. Witten, "Monopole Condensation, and Confinement in $\mathrm{N}=2$ Supersymmetric Yang-Mills Theory,” Nuclear Physics, Vol. 426, No. 1, 1994, pp. 19-52. doi:10.1016/0550-3213(94)90124-4 
[9] M. E. Peskin, "Dualities in Supersymmetric Yang-Mills Theories," hep-th/9703136.

[10] P. S. Bisht and O. P. S. Negi, "Revisiting Quaternionic Dual Electrodynamics," International Journal of Theoretical Physics, Vol. 47, No. 12, 2008, pp. 3108-3120. doi:10.1007/s10773-008-9744-8

[11] J. E. Avron, D. Osadchy and R. Seiler, “Topological Look at the Quantum Hall Effect,” Physics Today, Vol. 56, No. 8, August 2003, p. 38. doi:10.1063/1.1611351

[12] G. t' Hooft, "Magnetic, Monopoles in Unified Gauge Theories,” Nuclear Physics B, Vol. 79, No. 2, 1974, pp. 276-284.

doi:10.1016/0550-3213(74)90486-6 